Brit. Heart F., 1968, 30, 333.

\title{
Technique for Insertion of an Indwelling Intrapericardial Catheter
}

\author{
R. A. MASSUMI, J. C. RIOS, A. M. ROSS, AND G. A. EWY \\ From the George Washington University and Georgetown University Medical Divisions and Cardiopulmonary Labora- \\ tory, District of Columbia General Hospital, Washington D.C., 20003, U.S.A.
}

Needle aspiration of pericardial effusion is a universally accepted procedure both for relief of cardiac compression and for diagnostic laboratory studies. It is, however, performed with some trepidation in view of the possibility of coronary and myocardial injury and of arrhythmias. The probability of these risks is much greater in prolonged procedures aimed at evacuating the pericardial sac when frequent repositioning of the needle becomes necessary.

The purpose of this communication is to report a simple and safe technique for insertion into the pericardial sac of a soft, relatively large, radiopaque catheter permitting repeated aspirations and prolonged physiological observations. This should be of particular interest to thoracic surgeons confronted with rapidly reaccumulating traumatic haemopericardium, and to physiologists interested in the study of the dynamics of cardiac tamponade.

\section{TrChNique}

The procedure is identical with the Seldinger method for intra-arterial insertion of a catheter (Seldinger, 1953). The essential instruments (Fig. 1) consist of (1) an 18gauge, thin wall 6 inch $(15 \cdot 25 \mathrm{~cm}$.) ventricular puncture needle with a round, non-cutting tip and a sharp penciltip stylet, $\star$ (2) a 47 inch $(119 \mathrm{~cm}$.) stainless steel, 0.035 inch $(0.09 \mathrm{~cm}$.) spring-guide wire with flexible tip, (3) a 10 inch $(25.4 \mathrm{~cm}$.) long, radiopaque polyethylene catheter, 0.049 inch $(0.125 \mathrm{~cm}$.) in internal diameter with its tip tapered over a flame to an internal diameter of 0.035 inch $(0.09 \mathrm{~cm}$.) and armed with 4 to 6 side holes near the tip and (4) an adapter of appropriate size for connecting the catheter to the aspiration syringe.

The needle is inserted in the angle between the xiphoid process and the free margin of the left costal cartilage. Insertion is made through a small blade tip

\footnotetext{
Received June 19, 1967.

$\star$ Becton-Dickinson and Co., Rutherford, New Jersey. Catalog No. XT437LNR.
}

puncture and the needle is advanced cephalad, posteriorly, and slightly to the left, as in conventional pericardiocentesis. Attachment of the hub of the needle to the Wilson central terminal of an electrocardiograph, as advocated by Bishop, Estes, and McIntosh (1956), will serve to indicate proximity of the tip of the needle to, or actual contact with, the myocardium. Contact between operator's hands and the needle-wire assembly is through dry gloves in order to avoid accidental delivery of electrical current to the myocardium causing ventricular fibrillation. After entering the pericardial cavity, the sharp stylet is removed and the guide wire is advanced through the needle, under fluoroscopic control when possible, for approximately 10 in. $(25.4 \mathrm{~cm}$.). The needle is then removed, and the tapered tip of the catheter is threaded over the guide wire and advanced until it reaches the skin at the point of entrance. The catheter-guide wire assembly is then advanced through the skin and subjacent tissues, following the path of the already inserted guide wire for about 6 in. $(15 \cdot 2 \mathrm{~cm}$.). The tip of the catheter now lies within the pericardial sac (Fig. 2). The guide wire is then removed and the catheter is ready to be attached to the aspiration syringe. Before evacuating the effusion, the catheter can be connected to a pressure transducer for the study of intrapericardial pressure and its fluctuations with respiration. The catheter can be advanced or retracted and aspiration can be continued or repeated as deemed necessary without further discomfort to the patient or risk of myocardial irritation.

\section{RESUlts}

This procedure, performed thus far in 12 patients with large effusions over the past 4 years, has not been associated with any complications. Large pericardial effusions were recovered in 10 patients. No fluid was obtained from only one patient with purulent pericarditis during pericardiocentesis, but large amounts were recovered at thoracotomy. Occlusion of the lumen of the catheter has not occurred. In three patients the procedure was carried out 333 


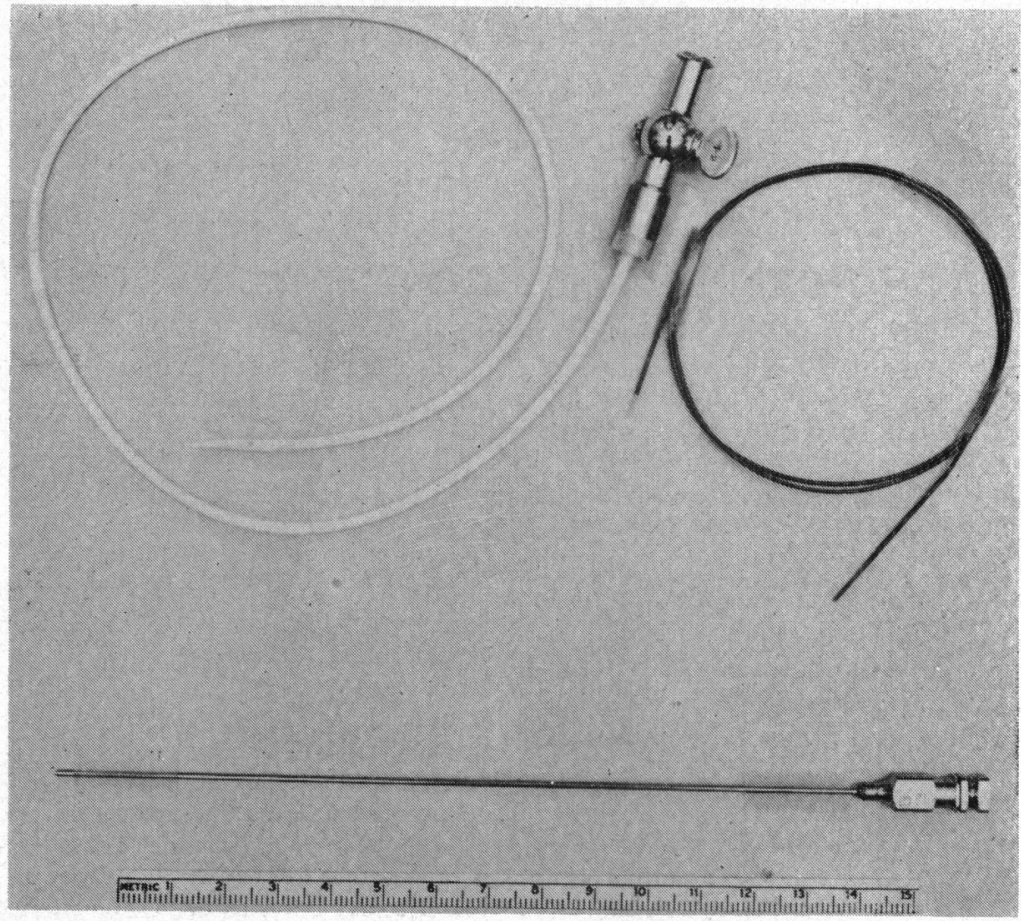

FIG. 1.-Instruments used for the insertion of a catheter into the pericardial sac. For descripton see text.

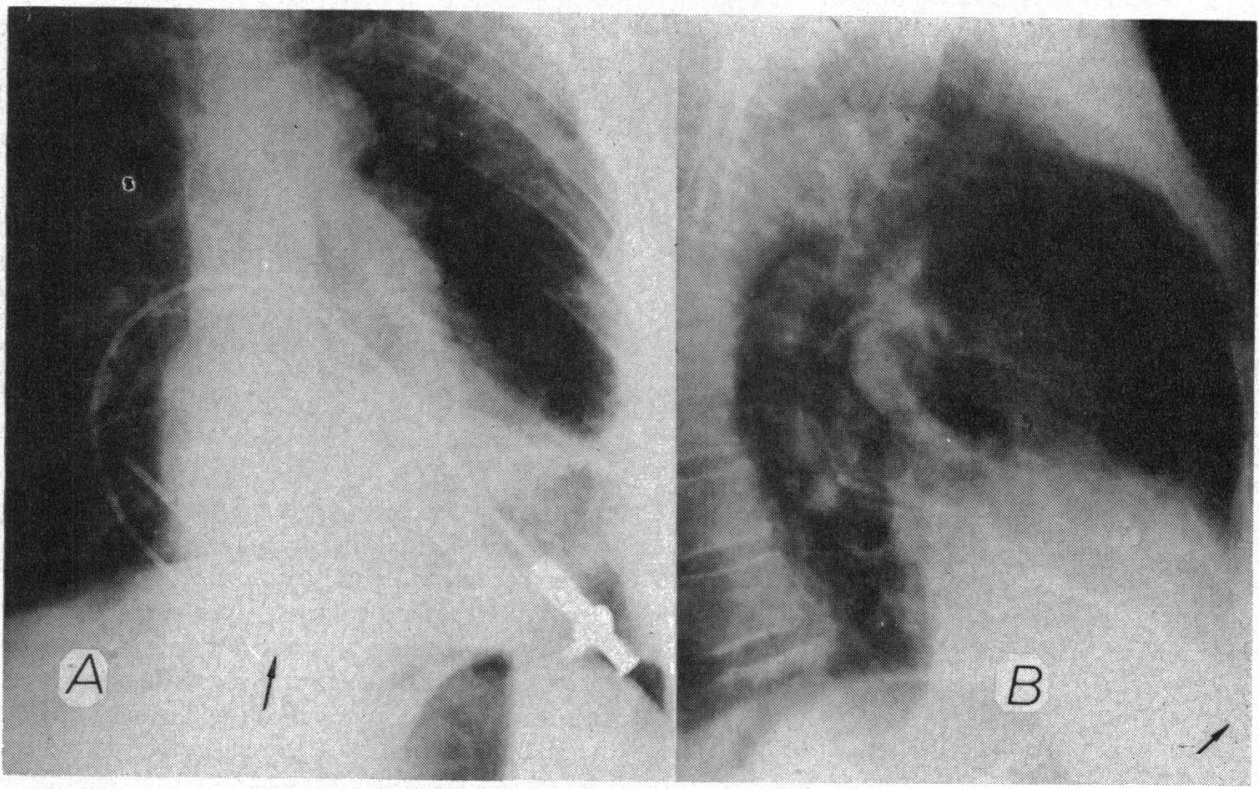

Fig. 2.-Chest $x$-ray film after insertion of an intrapericardial catheter. Arrows indicate point of entrance. 
place for 2 days on 2 occasions and for 7 days in 2 patients, without discomfort, detectable reactions, or occlusion of the holes. One patient had paroxysmal atrial fibrillation while the catheter was in place. This, however, was probably unrelated to the pericardiocentesis, as it appeared spontaneously on two other occasions. Furthermore, supraventricular arrhythmias are known to represent a frequent complication during the course of acute pericarditis (James, 1962) and were encountered in 3 patients in the present series. The physiological studies on the mechanism of cardiac tamponade, which have been made possible by this technique, are the subject of a separate communication.

\section{Discussion}

The technique of catheter insertion into the pericardial cavity eliminates all the dangers inherent in maintaining a rigid needle in the pericardial sac during the period of minutes to hours required for complete evacuation of large pericardial effusions. The initial introduction of the needle is identical with that of routine pericardiocentesis, and, as such, is recognized to be a relatively benign procedure. The problems with the latter technique arise, however, when the pericardial effusion diminishes in quantity and the tip of the needle approaches the myocardial surface. Failure to draw effusion through the needle as the tap proceeds necessitates repositioning or changes in direction of the needle, both of which are often complicated by ventricular arrhythmias, pneumothorax and by lacerations of the myocardium and occasionally of the coronary arteries (Hurst and Logue, 1966).

The total length of time the catheter can be left in place in the pericardial sac without provoking untoward reactions is unknown at this time. In our experience, the catheter has been left in place for 7 days both for removal of reaccumulating effusion and for the instillation of anti-neoplastic chemotherapeutic agent. After completion of therapy and cessation of effusion, the catheter is removed.

Asepsis is essential not only during the procedure but for the entire period when the catheter is indwelling. The point of entrance must be carefully dressed and kept dry and thoroughly clean so that bacteria may not reach the pericardial cavity along the outer surface of the catheter. The hub of the catheter and the adapters must be covered at all times, and further manipulation must be carried out by qualified personnel using aseptic techniques and sterile gloves.

The differences between this technique and those described by Schaffer (1959) and Livesley and Jack (1965) are that in the latter, a larger needle is required for the pericardiocentesis and that the catheter does not have side holes, thus making occlusion more likely.

\section{SUMMARY}

A simple technique for percutaneous introduction of a large pliable polyethylene catheter into the pericardial sac has been described. The technique carries no additional risks and possesses distinct advantages over the needle method. It allows complete evacuation of effusion and, in addition, makes possible haemodynamic observations over long periods. It is a particularly useful safety measure in rapidly reaccumulating effusions and for intrapericardial instillation of various therapeutic agents.

\section{REFERENCES}

Bishop, L. H., Estes, E. H., and McIntosh, H. D. (1956). The electrocardiogram as a safeguard in pericardiocentesis. F. Amer. med. Ass., 162, 264.

Hurst, J. W., and Logue, R. B. (1966). The Heart. McGraw Hill, New York.

James, T. N. (1962). Pericarditis and the sinus node. Arch. intern. Med., 110, 305.

Livesley, B., and Jack, G. D. (1965). Chronic pericardial effusion with cholesterol crystals. A case-report together with the description of a simple and safe technique for pericardial aspiration. Lancet, 1, 677.

Schaffer, A. I. (1959). Pericardiocentesis with the aid of a plastic catheter and ECG monitor. Amer. F. Cardiol., 4, 83.

Seldinger, S. I. (1953). Catheter replacement of the needle in percutaneous arteriography. Acta radiol. (Stockh.), 39, 368. 